\title{
A Lagrangian solution to the relationship between source strength and concentration profile under conditions of local advection
}

\author{
Guowang Qiu • Jon S. Warland
}

Received: 14 February 2006 / Accepted: 15 May 2006 /

Published online: 12 July 2006

CSpringer Science+Business Media B.V. 2006

\begin{abstract}
We propose a two-dimensional Lagrangian analytical solution for relating source strength and concentration profiles within and above a plant canopy. The new solution describes passive scalar dispersion under conditions of local advection through a fetch correction function in a one-dimensional Lagrangian analytical dispersion model. The model is capable of predicting absolute concentration profiles of passive scalars for different fetches for situations in which the reference concentration is known or the background concentration is available. Tests of the model showed good agreement with measurements from field and wind-tunnel experiments.
\end{abstract}

Keywords Fetch · Lagrangian model · Local advection · Plant canopy

\section{Introduction}

The relationship between sources/sinks and concentration profiles within and above plant canopies has been studied for many years, and it continues to be an important and active research subject in micrometeorology. Various models have been developed to describe this relationship above surfaces or within canopies based on turbulent transfer and dispersion theory. Early studies relied extensively on gradient diffusion theory, which has been questioned because of counter-gradient transport within canopies (Denmead and Bradley 1985; Raupach 1988). Other approaches, such as higher-order closure models, stochastic Lagrangian models and large-eddy simulation (LES) techniques, have emerged to overcome the limitations of gradient diffusion theory. The major disadvantage of these approaches is their large computational time requirements.

G. Qiu (凶) · J. S. Warland

Department of Land Resource Science, University of Guelph, Guelph, Ontario,

Canada N1G 2W1

e-mail: jwarland@uoguelph.ca 
As an alternative, Raupach (1989a) developed an analytical Lagrangian solution for relating scalar concentration profiles to source strength profiles based on 'localized near-field' theory (LNF), in which the scalar concentration was decomposed into near-field and far-field contributions and calculated separately. Simulated results showed that LNF is capable of capturing the counter-gradient fluxes within canopies, and provided comparable simulation ability to Lagrangian stochastic models (Raupach 1989a). Based on Taylor's (1921) dispersion theory, Warland and Thurtell (2000) (hereafter WT) developed an analytical solution to the relationship between scalar concentration profiles and sources inside plant canopies through a 'dispersion matrix'. Unlike LNF theory, the WT model uses turbulence statistics at both source height and the height of interest to describe the dispersion matrix, and calculates mean concentration contributed from both near-field and far-field. Their simulated results showed better agreement than LNF with measured temperature profiles from a wind-tunnel heat dispersion experiment (Coppin et al. 1986). Both models, however, were developed only for horizontally homogeneous, one-dimensional situations.

The objective of the present study was to develop a two-dimensional analytical Lagrangian solution to the relationship between scalar concentration profiles and source distributions within and above a canopy. In contrast to the LNF and WT models, the present solution allows for horizontal inhomogeneity of the tracer field, though it was derived based on the solution for homogeneous turbulence. Such a solution is useful for a variety of applications ranging from flux footprint prediction to gas exchange estimation. As with the model of Lee (2004) for scalar advection inside canopies, this solution can also be used for footprint investigation. Compared to Lee's model in which the numerical procedure was used to solve the equations, the significant advantage of this solution is that it is analytical and therefore simple and suitable for daily use. Further, this solution can be used to predict absolute concentration profiles for different fetches from ground and canopy sources in which the reference concentration is known or the background concentration is available. The mathematical derivation of the model is reported in Section 2, and Section 3 describes tests of the model with available data.

\section{Derivation of model}

The derivation begins with a Lagrangian analysis of turbulent dispersion, in which the vertical gradient from a plane source is derived from the solution for a line source in a uniform flow with homogeneous turbulence. Comparing this vertical gradient to the one-dimensional WT model for near-field and far-field, a fetch correction function is proposed. Following WT, it is assumed that the canopy is composed of several thin horizontal layers (planes) stacked on top of each other, and the concentration gradient at any height is the sum of the gradients produced by the sources of each layer. This enables us to calculate the gradient, and consequently the concentration profile from the source layers (canopy source). The derivation of this two-dimensional model is based on a uniform flow with homogeneous turbulence, and assumes that: (i) inhomogeneous turbulence in and above a canopy can be approximated using the homogeneous solution, and (ii) dispersion in a real flow can be approximated by dispersion in a uniform flow. This allows us to apply the model to describe turbulent scalar dispersion under advective conditions in and above a plant canopy. 
For fluid particles released from the origin in stationary, homogeneous turbulence, the standard deviation of particle displacement $\left(\sigma_{z}\right)$ at time $t$ is described by Taylor (1921)

$$
\sigma_{z}^{2}=2 \sigma_{w}^{2} \tau_{\mathrm{L}}\left[t-\tau_{\mathrm{L}}+\tau_{\mathrm{L}} \exp \left(-t / \tau_{\mathrm{L}}\right)\right],
$$

where $\sigma_{w}$ is the standard deviation of the vertical velocity, and $\tau_{\mathrm{L}}$ is the Lagrangian time scale. Equation 1 has limits:

$$
\begin{aligned}
& \lim _{t / \tau_{\mathrm{L}} \rightarrow 0} \sigma_{z}^{2}=\sigma_{w}^{2} t^{2}, \\
& \lim _{t / \tau_{\mathrm{L}} \rightarrow \infty} \sigma_{z}^{2}=2 \sigma_{w}^{2} \tau_{\mathrm{L}} t .
\end{aligned}
$$

Equation 2 shows that the dispersion of particles produces a cloud depth increasing linearly with time when $t / \tau_{\mathrm{L}} \rightarrow 0$ (near field), while behaving as a classic diffusion process when $t / \tau_{\mathrm{L}} \rightarrow \infty$ (far field).

Assuming homogeneous turbulence in a uniform wind, the standard deviation of particle displacement after travel time $t=\left(x-x_{s}\right) / u$ (where $x_{s}$ is the location of the source along the $x$-axis and $u$ is the constant wind velocity in the $x$ direction) can also be expressed in terms of the travel distance $x-x_{s}$. Hence, Eq. 2 can be rewritten as

$$
\begin{aligned}
& \lim _{\frac{x-x_{s}}{u \tau_{\mathrm{L}}} \rightarrow 0} \sigma_{z}^{2}=\sigma_{w}^{2}\left(\left(x-x_{s}\right) / u\right)^{2}, \\
& \lim _{\frac{x-x_{s}}{u \tau_{\mathrm{L}}} \rightarrow \infty} \sigma_{z}^{2}=2 \sigma_{w}^{2} \tau_{\mathrm{L}}\left(x-x_{s}\right) / u .
\end{aligned}
$$

Let us first consider dispersion from a continuous infinite cross-wind line source located at $\left(x_{s}, z_{s}\right)$ with source strength $Q\left(\mathrm{~kg} \mathrm{~s}^{-1} \mathrm{~m}^{-1}\right.$ for mass, or $\mathrm{W} \mathrm{m}^{-1}$ for energy), in a homogeneous turbulent flow $\left(\sigma_{w}\right.$ and $\left.\tau_{\mathrm{L}}\right)$ with uniform wind velocity $(u)$, so that the concentration at $(x, z)$ downwind of the line source can be described by (following Csanady 1973; Arya 1999)

$$
C(x, z)=\frac{Q}{\sqrt{2 \pi} u \sigma_{z}} \exp \left[-\frac{\left(z-z_{S}\right)^{2}}{2 \sigma_{z}^{2}}\right],
$$

where $\sigma_{z}$ is a function of $x-x_{s}$. To simplify the derivation, Eq. 4 and the following derivation do not consider a boundary condition at the surface $(z=0)$, however a reflecting boundary is introduced in the application of this model. Equation 4 is a direct solution to the advection-diffusion equation with constant diffusivity $K$ and constant advecting windspeed $u$ :

$$
u \frac{\partial C}{\partial x}=K \frac{\partial^{2} C}{\partial z^{2}}
$$

with $\sigma_{z}=2 K t$. Here it is assumed that horizontal diffusion is negligible.

An area/plane source can be treated as a continuum of line sources each with infinitesimal width $\delta x_{s}$. Thus the equivalent areal source is given by $q=Q / \delta x_{s}$. For the infinite cross-wind plane source, the concentration profile $c(x, z)$ at a downwind distance $x$ away from the leading edge is obtained by integrating Eq. 4 from 0 to $x$

$$
c(x, z)=\int_{0}^{x} \frac{q}{\sqrt{2 \pi} u \sigma_{z}} \exp \left(-\frac{\left(z-z_{s}\right)^{2}}{2 \sigma_{z}^{2}}\right) \partial x_{s},
$$


where $q$ is the area source $\left(\mathrm{kg} \mathrm{s}^{-1} \mathrm{~m}^{-2}\right.$ for mass, or $\mathrm{W} \mathrm{m}^{-2}$ for energy), which satisfies the following condition:

$$
\begin{gathered}
q=q\left(z_{s}\right) \text { for } x \geq 0, \\
q=0 \text { for } x<0 .
\end{gathered}
$$

The vertical gradient of concentration is then given by differentiating Eq. 6

$$
\frac{\partial c(x, z)}{\partial z}=\frac{\partial}{\partial z}\left[\int_{0}^{x} \frac{q}{\sqrt{2 \pi} u \sigma_{z}} \exp \left(-\frac{\left(z-z_{s}\right)^{2}}{2 \sigma_{z}^{2}}\right) \partial x_{s}\right] .
$$

Since the derivative of the integral is mathematically the same as the integral of the derivative $\left(\frac{\partial}{\partial z} \int_{0}^{x} f\left(x_{s}, z\right) \partial x_{s}=\int_{0}^{x} \frac{\partial}{\partial z}\left[f\left(x_{s}, z\right)\right] \partial x_{s}\right)$, Eq. 8 can be rewritten as

$$
\frac{\partial c(x, z)}{\partial z}=-\int_{0}^{x} \frac{q}{\sqrt{2 \pi} u \sigma_{z}} \frac{\left(z-z_{s}\right)}{\sigma_{z}^{2}} \exp \left(-\frac{\left(z-z_{s}\right)^{2}}{2 \sigma_{z}^{2}}\right) \partial x_{s} .
$$

Substituting the near-field and far-field limits of $\sigma_{z}$ of Eq. 3 into Eq. 9 gives

$$
\left.\frac{\partial c(x, z)}{\partial z}\right|_{\mathrm{NF}}=-\int_{0}^{x} \frac{q u^{2}\left(z-z_{s}\right)}{\sqrt{2 \pi} \sigma_{w}^{3}\left(x-x_{s}\right)^{3}} \exp \left(-\frac{u^{2}\left(z-z_{s}\right)^{2}}{2 \sigma_{w}^{2}\left(x-x_{s}\right)^{2}}\right) \partial x_{s}
$$

for $\frac{x-x_{s}}{u \tau_{\mathrm{L}}} \rightarrow 0$

$$
\left.\frac{\partial c(x, z)}{\partial z}\right|_{\mathrm{FF}}=-\int_{0}^{x} \frac{q \sqrt{u}\left(z-z_{s}\right)}{4 \sqrt{\pi} \tau_{\mathrm{L}}^{3 / 2}\left(x-x_{s}\right)^{3 / 2} \sigma_{w}^{3}} \exp \left(-\frac{u\left(z-z_{s}\right)^{2}}{4 \sigma_{w}^{2} \tau_{\mathrm{L}}\left(x-x_{s}\right)}\right) \partial x_{s}
$$

for $\frac{x-x_{s}}{u \tau_{\mathrm{L}}} \rightarrow \infty$

where $u, \sigma_{w}$ and $\tau_{\mathrm{L}}$ are assumed constant with height.

Integrating Eq. 10 with respect to $x_{s}$ gives

$$
\begin{gathered}
\left.\frac{\partial c(x, z)}{\partial z}\right|_{\mathrm{NF}}=\frac{-q}{\sqrt{2 \pi} \sigma_{w}\left|z-z_{s}\right|} \exp \left(-\frac{u^{2}\left(z-z_{s}\right)^{2}}{2 \sigma_{w}^{2} x^{2}}\right), \\
\left.\frac{\partial c(x, z)}{\partial z}\right|_{\mathrm{FF}}=\frac{-q}{2 \sigma_{w} L_{\mathrm{L}}} \operatorname{erfc}\left(\sqrt{\frac{u\left(z-z_{s}\right)^{2}}{4 \sigma_{w} L_{\mathrm{L}} x}}\right),
\end{gathered}
$$

where $L_{\mathrm{L}}=\sigma_{w} \tau_{\mathrm{L}}$ is the Lagrangian length scale, and erfc is the complementary error function.

Using the general relationship $\partial c(x, z) / \partial z=M q$, dispersion coefficients for the near-field and far-field can be written as

$$
M_{\mathrm{NF}}= \begin{cases}\frac{-1}{\sqrt{2 \pi} \sigma_{w}\left(z-z_{s}\right)} \exp \left(-\frac{u^{2}\left(z-z_{s}\right)^{2}}{2 \sigma_{w}^{2} x^{2}}\right) & \text { for } z>z_{s}, \\ \frac{1}{\sqrt{2 \pi} \sigma_{w}\left(z_{s}-z\right)} \exp \left(-\frac{u^{2}\left(z-z_{s}\right)^{2}}{2 \sigma_{w}^{2} x^{2}}\right) & \text { for } z<z_{s}\end{cases}
$$




$$
M_{\mathrm{FF}}= \begin{cases}\frac{-1}{2 \sigma_{w} L_{\mathrm{L}}} \operatorname{erfc}\left(\sqrt{\frac{u\left(z-z_{s}\right)^{2}}{4 \sigma_{w} L_{\mathrm{L}} x}}\right) & \text { for } z>z_{s}, \\ \frac{1}{2 \sigma_{w} L_{\mathrm{L}}} \operatorname{erfc}\left(\sqrt{\frac{u\left(z-z_{s}\right)^{2}}{4 \sigma_{w} L_{\mathrm{L}} x}}\right) & \text { for } z<z_{s} .\end{cases}
$$

As noted by Raupach (1988), the transition between the near-field and far-field regions occurs at a distance downwind of $\Delta x \sim u \tau_{\mathrm{L}}$ away from the emitting source. Hence, the near-field region is quite small, extending a horizontal distance of order $h_{\mathrm{c}}$ (the canopy height) from the source (Raupach 1988). In the near-field region, the dispersion can be approximated by its value in locally homogeneous turbulence provided the inhomogeneity is not too strong (Raupach 1989a), while the far-field concentration involves a diffusion/advective transport process. Following this logic, the far-field fetch term, $\operatorname{erfc}\left(\sqrt{\frac{u\left(z-z_{s}\right)^{2}}{4 \sigma_{w} L_{L} x}}\right)$ was used to account for the effect of advection on the concentration gradient at a downwind distance $(x)$. The following dispersion coefficient, $M(x)$, is therefore proposed to describe the vertical concentration gradient at downwind distance $(x)$ in advective situations as

$$
M(x)=M_{1} \operatorname{erfc}\left(\sqrt{\frac{u\left(z-z_{s}\right)^{2}}{4 \sigma_{w} L_{\mathrm{L}} x}}\right),
$$

where $M_{1}$ is the solution to the one-dimensional situation proposed by Warland and Thurtell (2000) as

$$
M_{1}= \begin{cases}\frac{-1}{2 \sigma_{w} L_{\mathrm{L}}\left[1-\exp \left(-\sqrt{\left.\left.\frac{\pi}{2} \frac{\left(z-z_{s}\right)}{L_{\mathrm{L}}}\right)\right]}\right.\right.} & \text { for } z>z_{s}, \\ \frac{1}{2 \sigma_{w} L_{\mathrm{L}}\left[1-\exp \left(-\sqrt{\frac{\pi}{2}} \frac{\left(z_{s}-z\right)}{L_{\mathrm{L}}}\right)\right]} & \text { for } z<z_{s} .\end{cases}
$$

As $x$ approaches infinity, the erfc function approaches 1 and Eq. 13 reduces to $M_{1}$, which is identical to the one-dimensional case.

To test Eq. 13, the dispersion coefficients were calculated from a numerical integration performed on Eq. 9 using the complete $\sigma_{z}$ expression (Eq. 1). The results are illustrated in Fig. 1 for different downwind distances, from which it can be seen that Eq. 13 is a close approximation of the continuous near-field/far-field dispersion under advective conditions described by Eq. 1 for different downwind distances $(x)$, but the error increases close to the upwind edge $(x \approx 0)$. It should be noted that, in the case of homogeneous turbulence, Eq. 13 is equal to Eq. 12 in the far-field limit for a ground-level source since the near-field contribution vanishes in this case.

Following a similar approach to WT, the concentration and gradient at a point are expressed in a layer with a mean value across the layer. The sources are specified in 

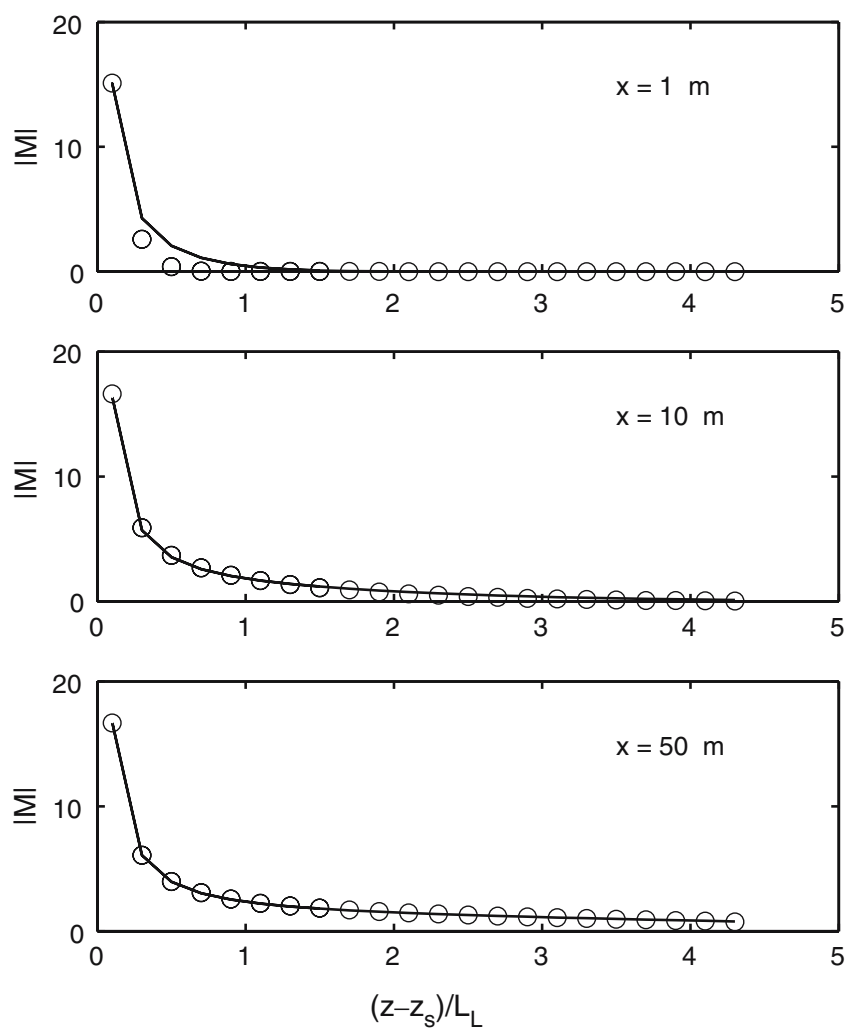

Fig. 1 Comparison of dispersion factor, $|M|$, from the numerical integration of Eq. 9 using full $\sigma_{z}$ (circles) and $|M|$ from Eq. 13 (line) as a function of $\left(z-z_{s}\right) / L_{\mathrm{L}}$ for different fetches $(x=1,10$ and $50 \mathrm{~m})$, with $z_{s}=0.85 \mathrm{~m}, \tau_{\mathrm{L}} u_{*} / h=0.4, \sigma_{w} / u_{*}=1.25$ and $u / u_{*}=10$

multiple layers, and assumed to be distributed evenly across the layer. The concentration gradient relates to the source strength through a dispersion matrix as

$$
\left.\frac{\mathrm{d} c(x, z)}{\mathrm{d} z}\right|_{i}=\sum_{j=1}^{m} M_{i j}(x) q_{j} \Delta z_{j}
$$

where $M_{i j}(x)$ is the dispersion matrix at the downwind distance $x, i$ and $j$ are concentration and source layer indices, respectively, $\Delta z_{j}$ is the thickness of the source layer, and $q_{j}$ has dimensions [ $\mathrm{M} \mathrm{L}^{-3} \mathrm{~T}^{-1}$ ]. Applying a reflecting boundary condition (e.g. Csanady 1973) and Warland and Thurtell's (2000) one-dimensional dispersion matrix $\left(M_{1 i j}\right)$, the dispersion matrix $M_{i j}(x)$ for two-dimensional situations in a uniform flow with homogeneous turbulence is

$$
M_{i j}(x)=M_{1 i j} \operatorname{erfc}\left(\sqrt{\frac{u\left(z_{i}-z_{j}\right)^{2}}{4 \sigma_{w} L_{\mathrm{L}} x}}\right) \text {, }
$$


where $M_{1 i j}$ is given by

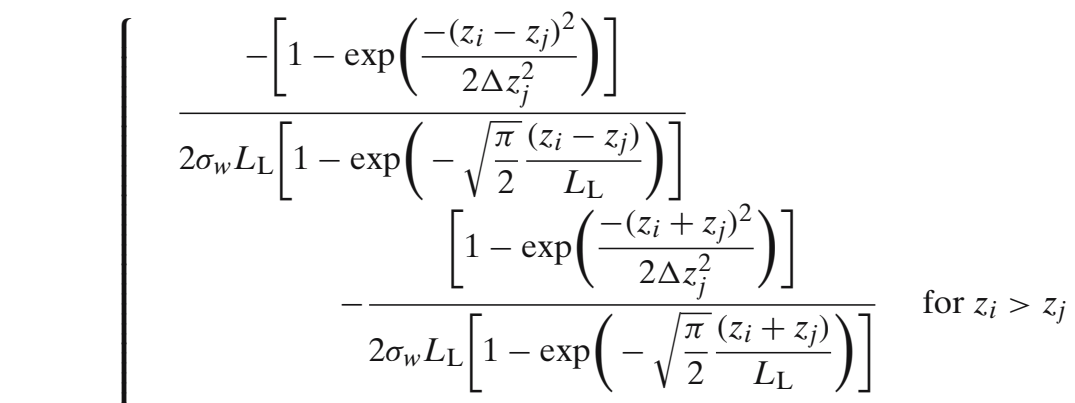

$$
\begin{aligned}
& \text { for } z_{i}=z_{j}
\end{aligned}
$$

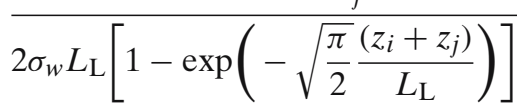

$$
\begin{aligned}
& \frac{\left[1-\exp \left(\frac{-\left(z_{i}-z_{j}\right)^{2}}{2 \Delta z_{j}^{2}}\right)\right]}{2 \sigma_{w} L_{\mathrm{L}}\left[1-\exp \left(-\sqrt{\frac{\pi}{2}} \frac{\left(z_{j}-z_{i}\right)}{L_{\mathrm{L}}}\right)\right]} \\
& -\frac{\left[1-\exp \left(\frac{-\left(z_{i}+z_{j}\right)^{2}}{2 \Delta z_{j}^{2}}\right)\right]}{2 \sigma_{w} L_{\mathrm{L}}\left[1-\exp \left(-\sqrt{\frac{\pi}{2}} \frac{\left(z_{i}+z_{j}\right)}{L_{\mathrm{L}}}\right)\right]} \quad \text { for } z_{i}<z_{j} .
\end{aligned}
$$

The vertical concentration profile at the specified downwind distance $(x)$ can be calculated from

$$
c_{i+1}(x)=c_{i}(x)+\frac{1}{2}\left(\left.\frac{\mathrm{d} c(x, z)}{\mathrm{d} z}\right|_{i}+\left.\frac{\mathrm{d} c(x, z)}{\mathrm{d} z}\right|_{i+1}\right)\left(z_{i+1}-z_{i}\right) .
$$

The above result can be applied directly to homogeneous turbulence, and the next step is to apply the model to conditions of inhomogeneous turbulence. Raupach (1989a) has shown that the homogeneous description is a reasonable approximation to weakly inhomogeneous turbulence, e.g. turbulence in plant canopies. Warland and Thurtell (2000) successfully applied their model to inhomogeneous turbulence with $\sigma_{w}$ replaced by its layer value $\sigma_{w i}$ and $L_{\mathrm{L}}$ replaced by the average value $\left(L_{\mathrm{L} i}+L_{\mathrm{L} j}\right) / 2$, where $j$ is the source layer. Numerical simulations and field tests of the LNF (Katul et al. 1997; Denmead et al. 2000; Harper et al. 2000; Leuning et al. 2000; Siqueira et al. 2000) and WT (Hsieh et al. 2003; Qiu et al. 2006) have confirmed that such an approximation is valid. 
A means to extend this solution from a uniform wind field to realistic wind profiles is still required for the model's application. There are three possible wind input options: (i) wind speed at the concentration layer (i), (ii) wind speed at the source layer $(j)$, or (iii) the mean wind speed between the concentration layer and the source layer. Here we propose to use the mean wind velocity between the concentration layer and source layer (which can be treated as an effective wind speed between source and concentration layers) because the transport occurs between the source layer and the concentration layer, similar to the solution of $L_{\mathrm{L}}$ in WT. The effective wind velocity for each layer is defined as

$$
U_{i}= \begin{cases}\frac{\frac{1}{2} \sum_{k=j+1}^{i}\left(u_{k}+u_{k-1}\right)\left(z_{k}-z_{k-1}\right)}{\sum_{k=j+1}^{i}\left(z_{k}-z_{k-1}\right)} & \text { for } z_{i}>z_{j}, \\ u_{i} & \text { for } z_{i}=z_{j}, \\ \frac{\frac{1}{2} \sum_{k=i+1}^{j}\left(u_{k}+u_{k-1}\right)\left(z_{k}-z_{k-1}\right)}{\sum_{k=i+1}^{j}\left(z_{k}-z_{k-1}\right)} & \text { for } z_{i}<z_{j} .\end{cases}
$$

For a ground source, Eq. 19 reduces to

$$
U_{i}=\frac{\frac{1}{2} \sum_{k=1}^{i}\left(u_{k}+u_{k-1}\right)\left(z_{k}-z_{k-1}\right)}{\sum_{k=1}^{i}\left(z_{k}-z_{k-1}\right)} .
$$

Horizontal inhomogeneity also exists under advective conditions. A reasonable solution is to use the mean values $\overline{\sigma_{w i}(x)}, \overline{L_{\mathrm{Li}}(x)}$ and $\overline{U_{i}(x)}$ between the leading edge $(x=0)$ and downwind distance $(x)$ as long as $\sigma_{w i}, L_{\mathrm{L} i}$ and $U_{i}$ as functions of $x$ are known. However, applying $\overline{\sigma_{w i}(x)}, \overline{L_{\mathrm{Li}}(x)}$ and $\overline{U_{i}(x)}$ to the model is not feasible when functions of $\sigma_{w i}(x), L_{\mathrm{L} i}(x)$ and $U_{i}(x)$ are unknown. As an alternative, we propose to use $\sigma_{w i}(x), L_{\mathrm{Li}}(x)$ and $U_{i}(x)$ at the downwind distance $(x)$ to replace $\overline{\sigma_{w i}(x)}, \overline{L_{\mathrm{L} i}(x)}$ and $\overline{U_{i}(x)}$ as an approximate solution if the streamwise variations of $\sigma_{w i}(x)$ and $L_{\mathrm{L} i}(x)$ and $U_{i}(x)$ are small, which is a modest criterion for many advective situations as discussed in Raupach (1989a).

This leads to the final form of the dispersion matrix for a two-dimensional situation

$$
M_{i j}(x)=M_{1 i j}(x) \operatorname{erfc}\left(\sqrt{\frac{U_{i}(x)\left(z_{i}-z_{j}\right)^{2}}{4 \sigma_{w i}(x) L_{\mathrm{L} i}(x) x}}\right),
$$

where $M_{1 i j}(x)$ for vertical inhomogeneous turbulence is given by WT as 


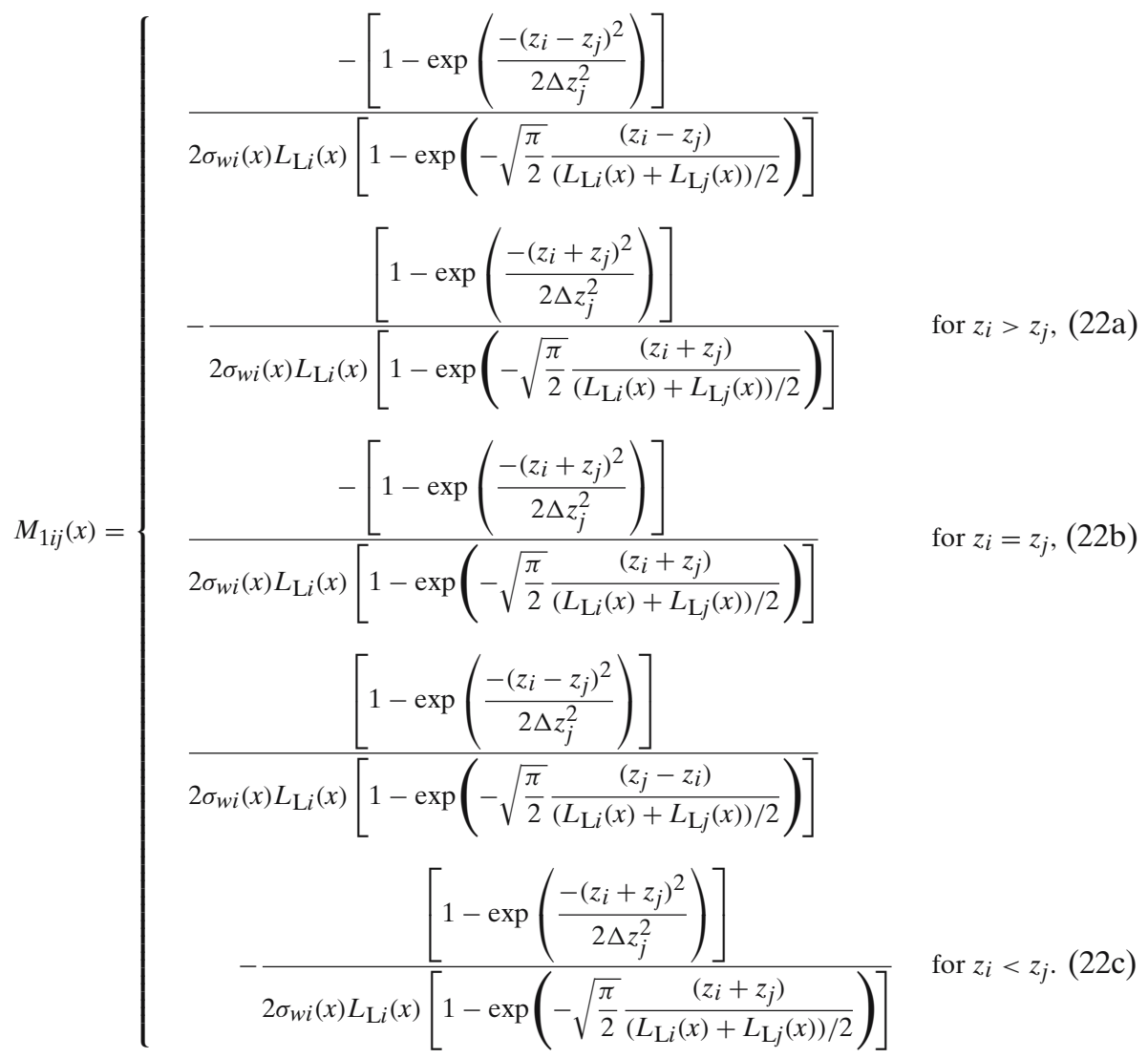

The model consists of Eqs. 15, 18, 21 and 22, with input parameters $\sigma_{w i}(x), L_{\mathrm{Li}}(x)$, $U_{i}(x)$ and $q_{j}(x)$ (or $\overline{\sigma_{w i}(x)}, \overline{L_{L i}(x)}, \overline{U_{i}(x)}$ and $\overline{q_{j}(x)}$ if they are available).

\section{Comparison of model with experimental data}

This section compares the model with the experimental data of Rider et al. (1963) for evaporation under advective conditions from a ground/surface source in the field, and of Coppin et al. (1986) for heat advection from a plane source in a wind-tunnel canopy.

\subsection{Comparison with ground/surface source}

Rider et al. (1963) reported an observational study of the variation in temperature and absolute humidity with distance and height from a dry-to-wet transition. This experiment was conducted at Canberra airport in Australia, and the profiles of temperature and humidity were measured from the tarmac/grass boundary to a downwind distance of $18.5 \mathrm{~m}$ in the grass area.

Because the original study did not report turbulence statistics, the following parameterizations were adopted. The standard deviation for vertical velocity under neutral conditions was specified as 


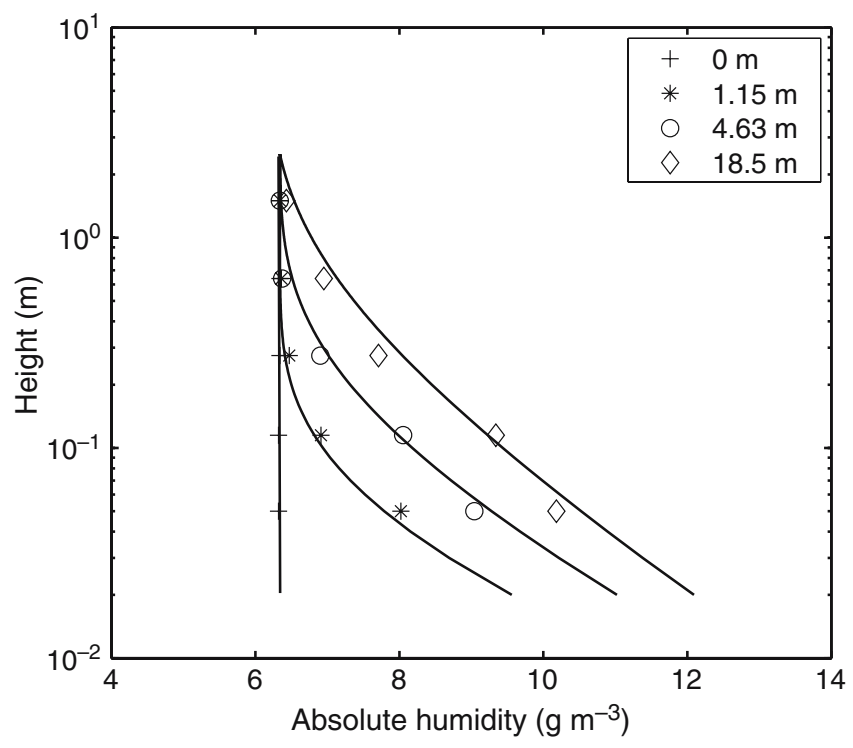

Fig. 2 Comparison of modelled absolute humidity profiles (lines) and experimental data (No. 6, Rider et al. 1963) for different downwind distances $(x)$

$$
\frac{\sigma_{w}}{u_{*}}=1.25,
$$

where $u_{*}$ is the friction velocity. The Lagrangian length scale above the surface under neutral conditions was parameterized as

$$
L_{\mathrm{L}}=0.5 z
$$

according to Wilson et al. (1981).

Profiles of $u$ for different downwind distances were digitized from Fig. 3 of Rao et al. (1974). Profiles of $\sigma_{w}$ and $L_{\mathrm{L}}$ were assumed to be constant with downwind distance, and the mean water vapour source strengths were calculated based on Fig. 7 of Rao et al. (1974). The background humidity well above the surface $(z=2.5 \mathrm{~m})$ was used to calculate the absolute humidity profiles at different downwind distances based on the predicted gradient profiles.

Figure 2 compares the model predictions of absolute humidity profiles with actual observations at various downwind distances from the leading edge (Rider et al. 1963). Modelled and measured humidities agree well in both profile shape and magnitude for various downwind distances. This is not surprising since the model is equal to the far-field limit solution for the ground source, provided that the two assumptions (made in Section 2) are valid in this case. The model captured the humidity profiles for various fetches even though the same $\sigma_{w}$ and $L_{\mathrm{L}}$ profiles were used for different fetches in this simulation.

To investigate the effect of the choice of wind profile on the humidity profile simulation, we repeated the absolute humidity profile calculations for various downwind distances using two wind profiles: (i) at $x=0 \mathrm{~m}$, and (ii) at $x=18.5 \mathrm{~m}$. The results are plotted in Fig. 3, along with the modelled humidity profiles using their corresponding wind profiles at different distances. Figure 3 shows that the modelled absolute 


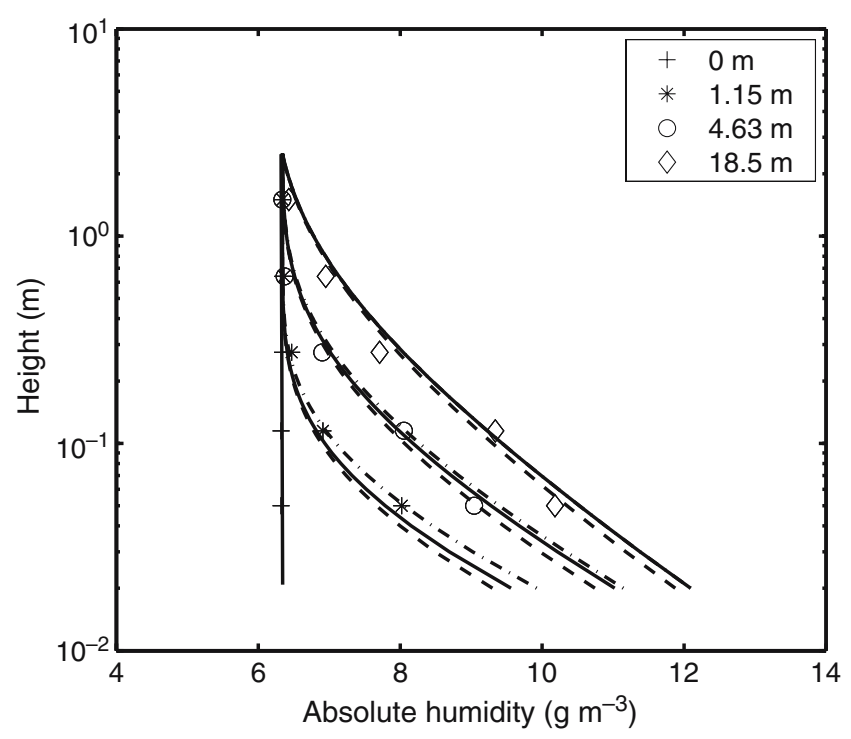

Fig. 3 The effect of wind profiles on model results. Lines are modelled downwind humidity profiles using different wind profile options (solid line: $u=u(x), x=1.15,4.63$ and $18.5 \mathrm{~m}$; dot-dash line: $u=u(x), x=18.5 \mathrm{~m}$; and dash line: $u=u(x), x=0 \mathrm{~m})$. Symbols $+, *, \circ$ and $\diamond$ represent observed humidity profiles at different fetches

humidity profiles were insensitive to horizontal inhomogeneity in $\bar{u}$. In the experiment of Rider et al. (1963), the horizontal gradients in $\bar{u}(x, z)$ were not very strong (Fig. 3 of Rao et al. 1974). The difference between upwind velocity $(x=0 \mathrm{~m})$ and downwind velocity $(x=18.5 \mathrm{~m})$ was less than $1.5 \mathrm{~m} \mathrm{~s}^{-1}$ near the ground $(z=0.015 \mathrm{~m})$ and decreased to $0.5 \mathrm{~m} \mathrm{~s}^{-1}$ at a height of $0.15 \mathrm{~m}$, implying that the insensitivity of the humidity profile to horizontal inhomogeneity may not hold under conditions of strong inhomogeneity. This also explains why the model works well though the same turbulence statistics for different fetches were used. However, the good agreement between modelled and measured humidity profiles suggests that it is valid to apply this model to inhomogeneous flow, at least for small streamwise flow variations.

\subsection{Comparison with in-canopy source}

Coppin et al. (1986) reported a wind-tunnel study on the dispersion of heat from a planar source within a plant canopy, conducted in the CSIRO Pye Laboratory wind tunnel in Australia. The canopy consisted of $60 \mathrm{~mm}$ tall metal strips spaced in a regular diamond array, with the planar heat source located at $z / h_{\mathrm{c}}=0.8$, where $h_{\mathrm{c}}$ is the canopy height. The heat source strength was $275 \mathrm{~W} \mathrm{~m}^{-2}$, and the temperature profiles were digitized from Coppin et al. (1986) for different fetches. The profiles of $\bar{u}$ and $\sigma_{w} / u_{*}$ for the above wind-tunnel experiment were reported by Raupach et al. (1986) and used in this calculation.

Since the measured Eulerian length scales in this wind-tunnel experiment were larger than those of other experiments (Raupach et al. 1986), the Lagrangian time scale was parameterized in this calculation following Leuning et al. (2000) for neutral conditions. The parameterized non-dimensional Lagrangian time scale is plotted in 


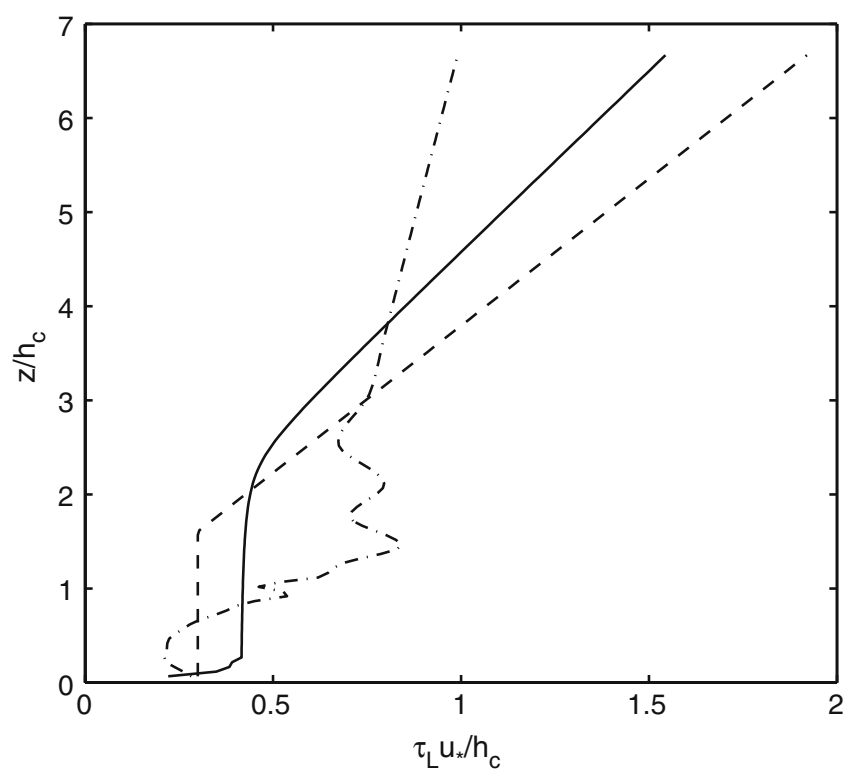

Fig. 4 Non-dimensional Lagrangian time scale as a function of normalised height. Solid, dash and dotand-dash lines indicate non-dimensional Lagrangian time scale parameterized according to Leuning et al. (2000), Raupach (1989b) and measured values (Raupach et al. 1986), respectively

Fig. 4, along with the measured values, and for comparison, Fig. 4 also shows the parameterized Lagrangian time scale adopted by Raupach (1989b). Model results using the above three length scale options were calculated. Results using the measured Lagrangian length scale and the one adopted by Raupach (1989b) were less satisfactory (result not shown), and so, the parameterized length scale of Leuning et al. (2000) was used in the following calculations.

To calculate absolute temperature profiles for different fetches, the background temperature $(\theta=0)$ well above the canopy $\left(z / h_{\mathrm{c}}=6.7\right)$ was used as the reference temperature. The calculated temperature profiles are plotted in Fig. 5, along with measured temperature profiles for various downwind distances from the leading edge $(x=0.53,1.06,1.54$ and $2.03 \mathrm{~m})$. The temperature was scaled by $\theta_{*}$, defined as

$$
\theta_{*}=\frac{H_{s}}{\rho c_{p} u_{*}},
$$

where $H_{s}$ is the plane source strength, $\rho$ is the air density and $c_{p}$ the specific heat of air at constant pressure.

In the upper canopy (above the source) and higher, the present model shows very good agreement with the wind-tunnel measurements in profile shape and magnitude for different fetches. It is understandable, since well above the canopy $\left(z / h_{c} \geq 2\right)$, the far-field dispersion dominates the overall dispersion from an extensive source (for example the canopy source), and our model captures far-field dispersion very well, not only for long fetches, but also for short fetches (Fig. 1).

The model underestimates temperature in the lower canopy (below the source) for shorter fetches $\left(x / h_{\mathrm{c}}=8.8,17.7\right.$ and 25.7), and the magnitude of error increases as the fetch decreases, though the shape of the predicted profiles is similar to the 
Fig. 5 Comparison of wind-tunnel data (Coppin et al. 1986) and the modelled temperature profiles from the present model. Canopy height $\left(h_{\mathrm{c}}\right)$ was $0.06 \mathrm{~m}$, the heat source strength was $275 \mathrm{~W} \mathrm{~m}^{-2}$, and located at $0.8 h_{\mathrm{c}}$. Symbols are observed values of temperature at various distances from the leading edge of the canopy (triangles, $x / h_{\mathrm{c}}=8.8$; asterisks, 17.7; stars, 25.7; and circles, 33.8). Lines represent modelled temperature profiles at the same distances as observations. Results from Warland and Thurtell's model are also shown (dash lines)

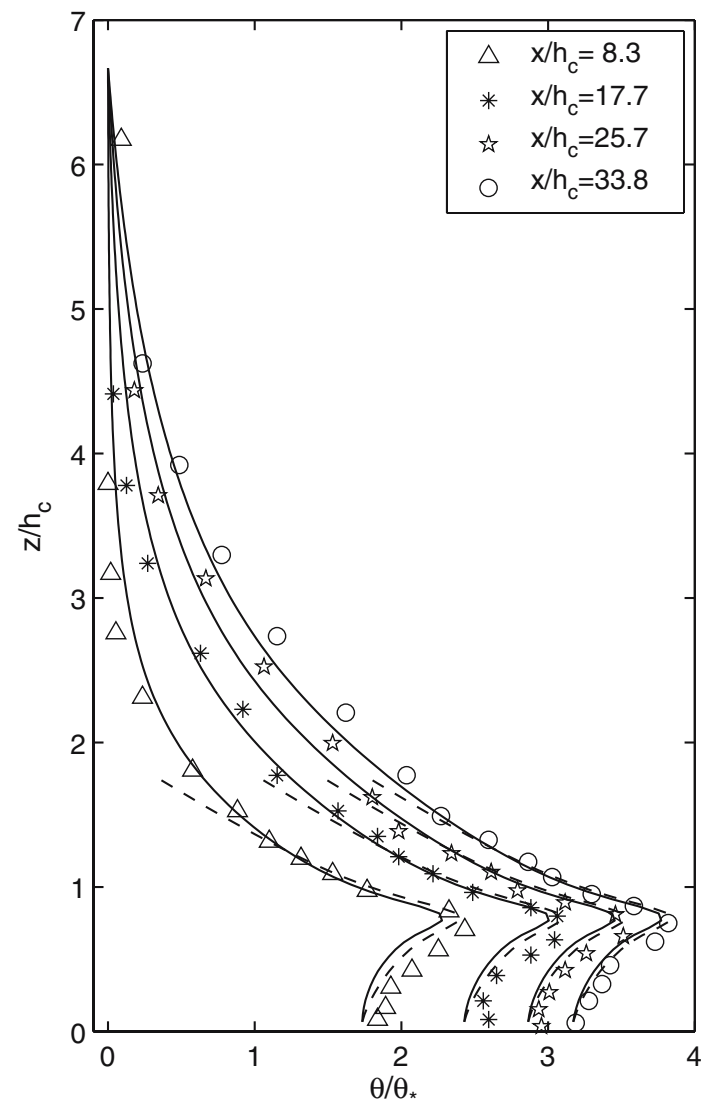

observed shape. The model works less well in the lower canopy for short fetches, and may be due to several factors. As shown in Fig. 1, the proposed dispersion matrix is an approximation of the continuous near-field/far-field dispersion under advective conditions, and the error will increase close to the leading edge, especially for the near-field calculation. Thus the poorer agreement between the modelled and measured mean temperature profiles for short fetches may be partly attributed to the errors introduced by the proposed dispersion matrix. In addition, the concentration profile was computed from the highest point based on the gradients, and so an error in one point could also affect all the calculations below it (Warland and Thurtell 2000).

Lee (2004) reported a mathematic model for scalar advection inside canopies, and the model was also tested using data from the Coppin et al. (1986) wind-tunnel heat dispersion experiment. In general, the two models (this model and Lee's model) show similar good overall agreement with the wind-tunnel measurements. Above the canopy (where the far-field diffusion dominates), the present model achieves better agreement than Lee's model. However, within the canopy (where the near-field dispersion dominates), Lee's model produces a more pronounced near-field effect in this canopy, especially close to the source height.

Figure 5 also shows the one-dimensional profile predictions of WT using the modelled temperatures nearest the surface from the two-dimensional model as the 
reference temperatures. Both models (WT and this model) produce similar temperature profiles within and just above the canopy, but diverge above $z / h_{\mathrm{c}} \approx 1.25-1.5$. The slight difference between WT and this model for $z / h_{\mathrm{c}} \leq 1.5$ may be attributed to: (1) the temperature gradient being in streamwise equilibrium within and just above the canopy (Coppin et al. 1986); and (2) the near-field dispersion having a dominant influence on scalar concentrations in the canopy because the leaves nearest to any point in the canopy make the strongest contributions to the scalar concentrations there (Raupach 1988). Hsieh et al. (2003) also reported a similar result by comparing results from their two-dimensional Lagrangian stochastic dispersion model and WT. This suggests that the assumption of local horizontal homogeneity of flow statistics is satisfactory in the description of dispersion within a plant canopy, and could also explain why the assumption of constant $\bar{u}$ at the beginning of the derivation did not lead to unacceptable inaccuracies.

To calculate the concentration profile using WT, the concentration must be known or specified at one height (known as the reference concentration) because WT only predicts the profile shape for one-dimensional situations. Similar to WT, this model also predicts concentration gradients. However, because the gradient goes to zero with concentration well above a canopy under certain situations such as the wind-tunnel experiment, this model also allows predictions of absolute concentration profiles in these circumstances without knowing the reference concentration. In addition, this model predicts the shapes of concentration profiles for different fetches, which make it possible to calculate the concentration difference between different fetches, even though no reference or background concentration is available. This information is extremely useful, and can be used to investigate/assess the effect of advection on concentrations along downwind distances $(x)$. Compared to higher-order closure models and Lagrangian stochastic models, the distinct advantages of this analytical Lagrangian model are its small computational time requirements and relative simplicity.

\section{Conclusions}

In this study, an analytical Lagrangian solution to the relationship between source strengths and concentration profiles within and above canopies under conditions of local advection has been presented. The model accounts for the effect of advection on concentration gradients for different downwind distances, and compared to one-dimensional Lagrangian models (e.g. LNF and WT), a wind profile at the downwind distance is the only additional input variable required.

The model was derived under a uniform flow with homogeneous turbulence, but it was applied to weakly inhomogeneous turbulence, as previously done for other models (e.g. LNF and WT). Field tests of LNF and WT have demonstrated that this assumption is justified for weak inhomogeneous turbulence (inside canopies). The model agrees well with previously published data in the field (Rider et al. 1963) and in a wind tunnel (Coppin et al. 1986), and appears valid for both vertical and horizontal inhomogeneous flows, at least in the case of small streamwise variation of $\sigma_{w}, L_{\mathrm{L}}$ and $u$. Under this criterion, the $\sigma_{w}, L_{\mathrm{L}}$ and $u$ at fetch $x$ can be used to substitute for the corresponding mean values between the leading edge and fetch $x$.

It is interesting to note that this two-dimensional model and one-dimensional WT model produce similar concentration profiles within the canopy, but diverge above the canopy. This result suggests that the effect of advection on concentration profiles 
within canopies is small, which may be attributed to the dominant influence of the near-field (local homogeneity) on scalar concentrations in the canopy.

The model predicts the shapes of concentration profiles at different fetches, which can be used to assess the effect of advection on concentrations along the downwind distances, even though no reference or background concentration is available. In addition to predicting the shapes of concentration profiles for different fetches, this model is also capable of predicting absolute concentration profiles for the situation in which the reference concentration is known or the background concentration is available. However, further investigation of the streamwise variations of $\sigma_{w}, L_{\mathrm{L}}$ and $u$ and their effects on the model under field conditions is still needed.

Acknowledgements We would like to thank Dr. George Thurtell for helpful suggestions with regard to the tests of this model. Thanks to Dr. Xuhui Lee and the other anonymous reviewer for their comments. We would also like to thank the Natural Sciences and Engineering Research Council of Canada and the Canadian Foundation for Climate and Atmospheric Sciences for their support of this work.

\section{References}

Arya SP (1999) Air pollution meteorology and dispersion. Oxford University Press, New York, $310 \mathrm{pp}$.

Coppin PA, Raupach MR, Legg BJ (1986) Experiments on scalar dispersion within a model plant canopy. Part II: An elevated plane source. Boundary-Layer Meteorol 35:167-191

Csanady GT (1973) Turbulent diffusion in the environment. D. Reidel Publishing Company, Boston, MA, $248 \mathrm{pp}$.

Denmead OT, Bradley EF (1985) Flux-gradient relationships in a forest canopy. In: Hutchison BA, Hicks BB (eds) The forest-atmosphere interaction. D. Reidel Publishing Company, Dordrecht, Holland, pp 421-442

Denmead OT, Harper LA, Sharpe RR (2000) Identifying sources and sinks of scalars in a corn canopy with inverse Lagrangian dispersion analysis I. Heat. Agric Forest Meteorol 104:67-73

Harper LA, Denmead OT, Sharpe RR (2000) Identifying sources and sinks of scalars in a corn canopy with inverse Lagrangian dispersion analysis II. Ammonia. Agric Forest Meteorol 104:75-83

Hsieh CI, Siqueira M, Katul G, Chu CR (2003) Predicting scalar source-sink and flux distributions within a forest canopy using a 2-D Lagrangian stochastic dispersion model. Boundary-Layer Meteorol 109:113-138

Katul G, Oren R, Ellsworth D, Hsieh C, Phillips N (1997) A Lagrangian dispersion model for predicting $\mathrm{CO}_{2}$ sources, sinks, and fluxes in a uniform loblolly pine (Pinus Taeda L.) stand. J Geophys Res 102:9309-9321

Lee X (2004) A Model for scalar advection inside canopies and application to footprint investigation. Agric Forest Meteorol 127:131-141

Leuning R, Denmead OT, Miyata A, Kim J (2000) Source/sink distributions of heat, water vapor, carbon dioxide and methane in a rice canopy estimated using Lagrangian dispersion analysis. Agric Forest Meteorol 104:233-249

Qiu G (2006) Development and testing of lagrangian models of scalar dispersion in canopies. Ph.D. Thesis, University of Guelph, Guelph, ON, $121 \mathrm{pp}$.

Rao KS, Wyngaard JC, Cote OR (1974) Local advection of momentum, heat, and moisture in micrometeorology. Boundary-Layer Meteorol 7:331-348

Raupach MR (1988) Canopy transport processes. In: Steffen WL, Denmead OT (eds) Flow and transport in the natural environment. Springer-Verlag, New York, p 95-127

Raupach MR (1989a) A practical Lagrangian method for relating scalar concentration to source distributions in vegetation canopies. Quart J Roy Meteorol Soc 115:609-632

Raupach MR (1989b) Applying Lagrangian fluid mechanics to infer scalar source distributions from concentration profiles in plant canopies. Agric Forest Meteorol 47:85-108

Raupach MR, Coppin, PA, Legg BJ (1986) Experiments on scalar dispersion within a model plant canopy. Part I: The turbulence structure. Boundary-Layer Meteorol 35:21-52

Rider NE, Philip JR, Bradley EF (1963) The horizontal transport of heat and moisture: a micrometeorological study. Quart J Roy Meteorol Soc 89:507-531 
Siqueira M, Lai C-T, Katul G (2000) Estimating scalar sources, sinks, and fluxes in a forest canopy using Lagrangian, Eulerian, and hybrid inverse models. J Geophys Res 105:29475-29488

Taylor GI (1921) Diffusion by continuous movements. Proc Lond Math Soc 20:196-211

Warland JS, Thurtell G (2000) A Lagrangian solution to the relationship between a distributed source and concentration profile. Boundary-Layer Meteorol 96:453-471

Wilson JD, Thurtell GW, Kidd GE (1981) Numerical simulation of particle trajectories in inhomogeneous turbulence, III: comparison of predictions with experimental data for the atmospheric surface layer. Boundary-Layer Meteorol 21:443-463 